\title{
Probe-Based Multiplex Real-Time PCR as a Diagnostic Tool to Distinguish Distinct Fungal Symbionts Associated With Euwallacea kuroshio and Euwallacea whitfordiodendrus in California
}

\author{
Joseph D. Carrillo, ${ }^{1}$ Joey S. Mayorquin, ${ }^{1}$ Jason E. Stajich, ${ }^{1}$ and Akif Eskalen ${ }^{2, \dagger}$ \\ ${ }^{1}$ Department of Microbiology and Plant Pathology, University of California, Riverside, CA 92521 \\ ${ }^{2}$ Department of Plant Pathology, University of California, Davis, CA 95616
}

\begin{abstract}
California has been invaded by two distinct Euwallacea spp. that vector unique plant pathogenic symbiotic fungi on multiple hosts and cause Fusarium dieback. The objective of this study was to develop multiplex real-time quantitative PCR assays using hydrolysis probes targeting the $\beta$-tubulin gene to detect, distinguish, and quantify fungi associated with the polyphagous shot hole borer (PSHB; Euwallacea whitfordiodendrus, Fusarium euwallaceae, Graphium euwallaceae, and Paracremonium pembeum) as well as the Kuroshio shot hole borer (KSHB; Euwallacea kuroshio, Fusarium kuroshium, and Graphium kuroshium) from various sample types. Absolute quantification reaction efficiencies ranged

Qualitative detection using the real-time assays on artificially inoculated avocado shoot extracts showed more sensitivity compared with conventional fungal isolation from wood. All symbiotic fungi, except $P$. pembeum, from PSHB and KSHB female heads were detectable and quantified. Field samples from symptomatic Platanus racemosa, Populus spp., and Salix spp. across 17 of 26 city parks were positively identified as PSHB and KSHB through detection of their symbiotic fungi, and both were found occurring together on five trees from three different park locations. The molecular assays presented here can be utilized to accurately identify fungi associated with these invasive pests in California.
\end{abstract} from 88.2 to $104.3 \%$, with a coefficient of determination $>0.992$ and a limit of detection of 100 copies $\mu l^{-1}$ for all targets across both assays.
Keywords: ambrosia beetle, pathogen detection
Bark and wood-boring weevils from the Scolytidae subfamily include $>6,000$ species that are of considerable economic importance in both temperate and tropical forests (Beaver et al. 2014). The Xyloborini tribe within this subfamily contains xylomycetophagous ambrosia beetles that are currently invading multiple locations in the world (Beaver et al. 2014; CABI 2015; O’Donnell et al. 2016). Although ambrosia beetles are known to be associated with fungi (Beaver et al. 2014), the symbiotic fungi can be phytopathogenic in some ambrosia beetle-fungi systems (Eskalen et al. 2013; Hulcr and Dunn 2011; Mendel et al. 2012; Ploetz et al. 2013). Fusarium dieback (FD) is a vascular disease of hardwood trees in southern California, and it is part of a highly invasive pest-disease complex threatening the avocado industry, urban forests, and native wildlands. There have been two separate invasions of exotic ambrosia beetles in California commonly called polyphagous shot hole borer (PSHB) (Eskalen et al. 2013) and Kuroshio shot hole borer (KSHB) (Stouthamer et al. 2017), both carrying similar but distinct suites of phytopathogenic fungi (Freeman et al. 2013; Lynch et al. 2016; Na et al. 2018; O'Donnell et al. 2015). The beetles have a wide host range of more than 300 plant species that are attacked, with 64 of those being suitable hosts

\section{${ }^{\dagger}$ Corresponding author: A. Eskalen; aeskalen@ucdavis.edu}

Funding: This project was supported by California Avocado Commission grant CAC-66013-85, California Department of Food and Agriculture Specialty Crop Block Grant Program grant SCB16051, California Association of Nurseries and Garden Centers grant CANERS 86003, a fellowship from the Los Angeles Center for Urban Natural Resources and Sustainability, and U.S. Department of Agriculture (USDA) Animal and Plant Health Inspection Service grant USDA-APHIS-AP18PPQS\&T00C162.

*The $\boldsymbol{e}$-Xtra logo stands for "electronic extra" and indicates that one supplementary figure is published online.

The author(s) declare no conflict of interest.

Accepted for publication 10 July 2019.

(c) 2020 The American Phytopathological Society for beetle reproduction (https://ucanr.edu/sites/pshb/overview/Hosts), and it is a great concern for the region, because there are limited strategies available for management of the pests.

Reproductive hosts are at more risk (Eskalen et al. 2013), and the progression of FD becomes severe when beetles bore into the xylem and then, continue to proliferate and accumulate on the host (Hulcr and Stelinski 2017). In early stages of host infestation, signs of beetle attacks are visible as entry holes ( $\sim 0.85$-mm diameter), and symptoms can be seen through various host responses to the boring activity of the beetle, which include gumming, sapping, staining, and sugar exudation (Eskalen et al. 2013). The impact of the disease can be severe in some areas, such as in urban landscape, where PSHB infested one-third of California sycamore (Platanus racemosa) in Orange County public parks. This caused the removal of 1,262 trees, resulting in $\sim$ US $\$ 4$ million in tree removal costs (OC Parks 2017). In riparian areas, such as the Tijuana river valley, which is dominated by Salix spp. and Populus spp., KSHB infestation levels are estimated to be $>53 \%$ in the conservation area as of 2015 (Boland 2016). The beetles are often misidentified as other common benign bark beetles and ambrosia beetles producing similar symptoms on various dead or dying trees. Although beetles can be recovered from the host for identification, they are usually difficult to obtain, because they are generally protected deeper in the wood $(25$ to $100 \mathrm{~mm})$ of the tree or are not present at all depending on the age of the beetle gallery. The beetles tend to inoculate their symbiotic fungi, because they initiate galleries in apparently healthy trees; however, the fungi can still grow in plant tissues (Hofstetter et al. 2015) regardless of whether the beetle is present since the trees remain alive after attacks. Therefore, targeting the symbiotic fungi associated with both PSHB and KSHB can provide accurate detection of these pest-disease complexes in the variety of hosts that they colonize in California.

PSHB and KSHB are morphologically indistinguishable, but they have been formally described as Euwallacea whitfordiodendrus (Schedl 1942) and Euwallacea kuroshio Gomez and Hulcr using conventional PCR and Sanger sequencing of the cytochrome oxidase I locus (Gomez et al. 2018), and they can also be distinguished using rapid high-resolution melt curve analysis using real-time PCR targeting the region (Rugman-Jones and Stouthamer 2017). The phytopathogenic fungi associated with the two species also differ in this 
invaded region; PSHB has been described to be associated with Fusarium euwallaceae (S Freeman, Z Mendel, T Aoki \& O’Donnell), Graphium euwallaceae (M Twizeyimana, S C Lynch \& A Eskalen), and Paracremonium pembeum (S C Lynch \& Eskalen), whereas KSHB is associated with Fusarium kuroshium (F Na, J D Carrillo \& A Eskalen) and Graphium kuroshium (F Na, J D Carrillo \& A Eskalen). There have been efforts to develop multiplexed and conventional PCR assays to target the Fusarium spp. within the ambrosia Fusarium clade (AFC) associated with Euwallacea spp. (Kasson et al. 2013; Short et al. 2017), but no efforts have been made to detect other confirmed pathogenic fungi associated with PSHB and KSHB from recovered samples.

Several PCR-based molecular methods have been developed for detection of plant pathogens, and among these methods, hydrolysis probe-based real-time quantitative PCR (qPCR) assays are the among the most sensitive (Palacio-Bielsa et al. 2009; Postollec et al. 2011; Raso and Biassoni 2014). The internal transcribed spacer (ITS) region of ribosomal DNA (rDNA) is the most commonly used locus for identifying fungal pathogens (Glass and Donaldson 1995; Schoch et al. 2012; White et al. 1990). rDNA can be present in amounts $>100$ copies per genome and has been reported to increase detection sensitivity (Tellenbach et al. 2010). However, in previous studies (Kasson et al. 2013; Na et al. 2018; O'Donnell et al. 2015), the ITS region of AFC fusaria associated with Euwallacea spp. was not as informative in distinguishing species owing to a lack of polymorphic sites compared with other loci, such as translation elongation factor 1- $\alpha$ (TEF1- $\alpha)$, DNA-directed RNA polymerase II largest $(R P B 1)$, and second largest subunit $(R P B 2)$. For example, $F$. euwallaceae from PSHB and $F$. kuroshium from KSHB were previously found to be completely identical at the ITS region (Na et al. 2018). A target sequence that consists of a single-copy gene per haploid genome and has more polymorphic regions, such as the $\beta$ tubulin (TUB2) gene, has been shown to provide more reliable quantification of fungal DNA from different samples types in multiplex qPCR reaction conditions (Atallah et al. 2007; Billones-Baaijens et al. 2018; Pouzoulet et al. 2013). The ability to detect and quantify fungal DNA using qPCR has been previously developed and applied to detect and quantify plant pathogens from plant tissue (Li et al. 2006, 2009; Oliveira et al. 2002; Qu et al. 2011). Although real-time PCR technology has been applied to detect a variety of plant pathogens affecting woody tree species (Bodles et al. 2006; Chandelier et al. 2018; Heller and Keith 2018; Osman and Rowhani 2006; Pouzoulet et al. 2013), none have been developed to target fungal pathogens vectored by invasive Euwallacea spp. that are currently causing damage in a wide range of hosts.

The objectives of this study were to (i) develop unique sets of oligonucleotides and hydrolysis probes to target and detect $F$. euwallaceae, G. euwallaceae, and P. pembeum from PSHB as well as F. kuroshium and G. kuroshium from KSHB; (ii) optimize multiplex qPCR assays for each complex to qualitatively identify the fungal targets across multiple sample types, including DNA extracts from infested wood, female beetle heads, and pure fungal cultures recovered from various reproductive hosts; (iii) quantify fungal targets from artificially inoculated avocado wood and laboratory-reared PSHB and KSHB female heads; and (iv) elucidate the distribution of both complexes from wood samples of cottonwood, sycamore, and willow collected from field surveys in sympatric areas.

\section{Materials and Methods}

Fungal isolates. Multiple isolates of fungal species ( $F$. euwallaceae, F. kuroshium, G. euwallaceae, G. kuroshium, and P. pembeum) obtained from previous studies (Eskalen et al. 2013; Lynch et al. 2016; Na et al. 2018) were used in this study from a culture collection maintained in the laboratory of A. Eskalen at the University of California, Riverside. In addition, isolates of Fusarium solani, Fusarium keratoplasticum, Fusarium petrophilum, Fusarium oxysporum, Fusarium brasiliense, Alternaria alternata, Clonostachys rosea, Colletotrichum gloeosporoides, Neofusicoccum parvum, and Penicillium expansum used in this study (Table 1) were included to test PSHB-FD and KSHB-FD assays for specificity against nonambrosial fungi. Fungal isolates recovered from beetles used in this study were obtained from the heads of female beetles using methods described by Lynch et al. (2016), and isolates recovered from galleries in wood followed methods described by Eskalen et al. (2013) and $\mathrm{Na}$ et al. (2018). All other isolates were provided by the Agricultural Research Service Culture Collection under permit USDA-P526P-1702585. All isolates used in this study were maintained on potato dextrose agar (PDA; BD Difco) amended with $0.01 \%$ tetracycline hydrochloride grown at $25^{\circ} \mathrm{C}$ for $\leq 2$ weeks.

DNA extractions. For DNA extraction of fungi from pure cultures, mycelium was harvested from isolates maintained on PDA from $60 \times 15$ petri dishes. After the plates were fully colonized, mycelium was placed into sterile $1.5-\mathrm{ml}$ microcentrifuge tubes containing $25 \mu 1$ of AP1 buffer (Qiagen), frozen at $-80^{\circ} \mathrm{C}$, and macerated with a plastic pestle (Thermo Fisher Scientific). After the tissue was macerated, the DNeasy plant mini kit (Qiagen) manufacturer's protocol was used to extract DNA from the samples. All samples were suspended in $50 \mu \mathrm{l}$ of AE elution buffer, and DNA concentration was quantified using a Nanodrop 2000c (Thermo Fisher Scientific) and diluted to a concentration of $10 \mathrm{ng} / \mu \mathrm{l}$.

For DNA extraction from symptomatic woody tissue, samples were first lyophilized with a benchtop freeze dryer (Labconco FreeZone 2.5 ) at $-50^{\circ} \mathrm{C}$ under a 0.01 -mbar vacuum (Labconco Rotary Vane Vacuum Pump, 195 LPM, 230V) for $24 \mathrm{~h}$. Wood samples were then transferred to sterile $2 \mathrm{ml}$ stainless steel metal microcentrifuge tubes along with two sterile stainless steel beads (2-mm diameter), plugged with sterile cotton, and then, refrozen at $-80^{\circ} \mathrm{C}$ for 30 min. After they were frozen, samples were capped with sterile impact-resistant plastic plugs and bead beaten for $30 \mathrm{~s}$ at $4.0 \mathrm{~m} / \mathrm{s}$ using a FastPrep (MP Biomedicals). Resulting wood dust was used for DNA extraction using the DNeasy plant mini kit manufacturer's protocol (Qiagen). All samples were suspended in $50 \mu \mathrm{l}$ of AE elution buffer, and DNA concentration was quantified using a Nanodrop 2000c (Thermo Fisher Scientific).

For DNA extraction from female beetles, samples were surface sterilized for $10 \mathrm{~s}$ using $1 \mathrm{ml}$ of $70 \%$ ethanol followed by two water washes using $1 \mathrm{ml}$ of sterile deionized water. After they were washed, the beetle heads were separated from the rest of the body, placed in sterile $1.5-\mathrm{ml}$ Eppendorf tubes, frozen at $-80^{\circ} \mathrm{C}$ for $30 \mathrm{~min}$, and then, macerated with a plastic pestle (Thermo Fisher Scientific). After the tissue was macerated, the DNeasy plant mini kit (Qiagen) was used to extract DNA from the samples. All samples were suspended in $50 \mu \mathrm{l}$ of AE elution buffer, and DNA concentration was quantified using a Nanodrop 2000c (Thermo Fisher Scientific).

Primer and probe design. TUB2 sequences from target Fusarium spp. were obtained from available genomes (GenBank accession nos.: NHTE00000000 $=F$. euwallaceae, NKUJ00000000 $=F$. $k u r$ oshium, NIZV00000000 = Fusarium ambrosium, NKCL00000000 = Fusarium sp. AF-3, NKCK00000000 = Fusarium oligoseptatum, NKCJ00000000 $=$ Fusarium sp. AF-6, and NKCI00000000 $=$ Fusa rium sp. AF-8) and checked for specificity of the target region with the FASTA program (Pearson 2016) against available genomes using the -fasta36 command. Graphium spp. and Paracremonium spp. TUB2 regions were amplified using conventional PCR with the Bt1a and Bt1b primer pair (Glass and Donaldson 1995). Products were purified using ExoSAP-IT (Affymetrix) and then, sequenced in both directions at the Institute for Integrative Genome Biology, University of California, Riverside with corresponding primers used for PCR. Species-specific primer sets and Primetime hydrolysis probes were designed for each target species by aligning sequences of targets with sequences obtained from GenBank (Table 1) from closely related members for each genus with Clustal X (Thompson et al. 1997) to determine the presence of polymorphic regions in the TUB2 gene. Polymorphic regions in the fourth intronic region of the $T U B 2$ gene were selected, and melting temperatures and secondary structures of generated oligonucleotides were evaluated using Primer3 (v. 0.4.0) (Koressaar and Remm 2007; Untergasser et al. 2012). Each primer set was designed to have at least one singlenucleotide polymorphism (SNP), with one targeted on the $3^{\prime}$ end 
of the oligonucleotide sequence. Hydrolysis probes were designed with five SNPs for target Fusarium spp. and three SNPs for target Graphium spp. between the respective species of interest (Table 2). Candidate oligonucleotide sequences were also checked for specificity by using the FASTA program (Pearson 2016) against available AFC genomes as well as the BLAST (Altschul et al. 1990). Separate assays for the two invasive pest disease complexes PSHB-FD and KSHB-FD in California were developed using five different fluorophores selected for species-specific hydrolysis probes targeting fungi from the PSHB-FD complex, including FAM ( $F$. euwallaceae), Texas 615 (G. euwallaceae), and Cy5.5 (P. pembeum), whereas HEX (F. kuroshium) and Cy5 (G. kuroshium) were selected for the KSHB-FD complex assay. All hydrolysis probes had $5^{\prime}$ fluorophore and 3 ' Iowa Black dark quencher modifications, and only FAM and HEX hydrolysis probes had internal ZEN quenchers; all were synthesized by Integrated DNA Technologies (IDT). The oligonucleotide sequences, product length, and melting temperatures are provided in Table 2.

Multiplexed real-time PCR reaction, optimization, and validation. The multiplexed PCR reactions were carried out in $25-\mu 1$ reactions using $1 \times$ iQ Supermix (Bio-Rad), $200 \mathrm{~nm}$ of each primer set, $100 \mathrm{~nm}$ of each hydrolysis probe, and $1 \mu l$ of DNA template or nuclease free water (Thermo Fisher Scientific) for no template controls (NTCs). All PCR amplifications were done using a two-step protocol: $94^{\circ} \mathrm{C}$ for $3 \mathrm{~min}$ followed by 33 cycles of denaturation at $94^{\circ} \mathrm{C}$ for $15 \mathrm{~s}$ and annealing/extension at $60^{\circ} \mathrm{C}$ for $45 \mathrm{~s}$, with plate readings taking place after the annealing extension step in real-time runs. All qPCR reactions were done in triplicate using Hardshell 96-well PCR plates (Bio-Rad) or 0.2-ml eight-tube low-profile strips (Bio-Rad) compatible with the CFX96 Touch Real-Time PCR Detection System (Bio-Rad) and analyzed using CFX manager (v.3.1).

The optimal multiplexed annealing/extension conditions for all primer sets were determined to be $60^{\circ} \mathrm{C}$ using the conventional PCR thermal gradient option with a MyCycler (Bio-Rad) and end point analysis. Amplified products were separated by gel electrophoresis in a $2.5 \%$ agarose gel run with $0.5 \times$ Tris-boric acid-EDTA buffer, stained with SYBR Green (Invitrogen), and visualized under ultraviolet light. Specific products were validated by using DNA from type specimens (Table 1) of the five target species and

Table 1. Representative fungi from Euwallacea whitfordiodendrus and Euwallacea kuroshio and various hosts in California used in this study

\begin{tabular}{|c|c|c|c|c|c|c|c|}
\hline \multirow[b]{2}{*}{ Isolate } & \multirow[b]{2}{*}{ Species } & \multirow[b]{2}{*}{ Host } & \multirow[b]{2}{*}{ Location $^{\mathbf{a}}$} & \multirow[b]{2}{*}{ SHB $^{b}$ complex } & \multicolumn{3}{|c|}{ GenBank no.b } \\
\hline & & & & & $\beta$-tubulin & ITS & TEF1- $\alpha$ \\
\hline NRRL62626a,c & Fusarium euwallaceae & Persea americana & Los Angeles, CA & PSHB & KU171782 & - & - \\
\hline UCR4511 & F. euwallaceae & Platanus racemosa & Riverside, CA & PSHB & MK108959 & - & - \\
\hline UCR4920 & F. euwallaceae & Eucalyptus sp. & Lake Forest, CA & PSHB & MK108960 & - & - \\
\hline UCR4937 & F. euwallaceae & Salix lasiolepis & Lake Forest, CA & PSHB & MK108961 & - & - \\
\hline NRRL54723 ${ }^{\mathrm{a}}$ & F. euwallaceae & Persea americana & Glil Yam, Israel & PSHB & MK108962 & - & - \\
\hline UCR2974 & Graphium euwallaceae & Ricinus communis & Los Angeles, CA & PSHB & MK108968 & - & - \\
\hline UCR2977 & G. euwallaceae & Acacia floribunda & Los Angeles, CA & PSHB & MK108969 & - & - \\
\hline UCR2979 & G. euwallaceae & Quercus agrifolia & Los Angeles, CA & PSHB & MK108970 & - & - \\
\hline UCR2980 & G. euwallaceae & Persea americana & Los Angeles, CA & PSHB & MK108971 & - & - \\
\hline UCR2981 ${ }^{\mathrm{c}}$ & G. euwallaceae & Persea americana & Los Angeles, CA & PSHB & MK108972 & - & - \\
\hline UCR2983 ${ }^{c}$ & Paracremonium pembeum & Persea americana & Los Angeles, CA & PSHB & KU053056 & - & - \\
\hline UCR2989 & P. pembeum & Ricinus communis & Los Angeles, CA & PSHB & KU053062 & - & - \\
\hline UCR2990 & P. pembeum & Ricinus communis & Los Angeles, CA & PSHB & KU053063 & - & - \\
\hline UCR2992 & P. pembeum & Persea americana & Los Angeles, CA & PSHB & KU053065 & - & - \\
\hline UCR2993 ${ }^{c}$ & P. pembeum & Ricinus communis & Los Angeles, CA & PSHB & KU053066 & - & - \\
\hline CBS142642 $2^{\mathrm{c}, \mathrm{d}}$ & F. kuroshium & Platanus racemosa & El Cajon, CA & KSHB & MK108963 & - & - \\
\hline $\mathrm{UCR} 3644^{\mathrm{c}}$ & F. kuroshium & Platanus racemosa & El Cajon, CA & KSHB & MK108964 & - & - \\
\hline UCR3651 & F. kuroshium & E. kuroshio & Fallbrook, CA & KSHB & MK108965 & - & - \\
\hline UCR3653 & F. kuroshium & Persea americana & Bonsall, CA & KSHB & MK108966 & - & - \\
\hline UCR3654 & F. kuroshium & Persea americana & Bonsall, CA & KSHB & MK108967 & - & - \\
\hline CBS142643 $3^{\mathrm{c}, \mathrm{d}}$ & G. kuroshium & Persea americana & Fallbrook, CA & KSHB & MK108973 & - & - \\
\hline UCR $4594^{\mathrm{c}}$ & G. kuroshium & Persea americana & Fallbrook, CA & KSHB & MK108974 & - & - \\
\hline UCR4606 & G. kuroshium & Persea americana & Bonsall, CA & KSHB & MK108975 & - & - \\
\hline UCR4607 & G. kuroshium & Persea americana & Bonsall, CA & KSHB & MK108976 & - & - \\
\hline UCR4608 & G. kuroshium & Euwallacea kuroshio & Bonsall, CA & KSHB & MK108977 & - & - \\
\hline UCR7265 & F. petrophilum & Persea americana & Escondido, CA & N/A & - & MK101041 & MK818422 \\
\hline UCR7266 & F. petrophilum & Platanus racemosa & Irvine, CA & N/A & - & MK101040 & MK818420 \\
\hline NRRL13414 ${ }^{\mathrm{a}}$ & F. keratoplasticum & N/A & California, U.S.A. & N/A & - & MK806583 & MK818415 \\
\hline NRRL22253 ${ }^{a}$ & F. petrophilum & Prunus persica & Hebei, China & N/A & - & MK806584 & MK818414 \\
\hline NRRL22639 & F. solani & Allium сера & Canada & N/A & - & MK806585 & MK818416 \\
\hline UCR3424 & F. oxysporum & Persea americana & Irvine, CA & N/A & - & MK101043 & MK818419 \\
\hline UCR6846 & F. oxysporum & Persea americana & Riverside, CA & N/A & - & MK101042 & MK818421 \\
\hline NRRL38352 ${ }^{a}$ & F. oxysporum & Solanum tuberosum & Texas, U.S.A. & N/A & - & MK806587 & MK818418 \\
\hline NRRL38357a & F. oxysporum & Eustoma sp. & Florida, U.S.A. & N/A & - & MK806588 & MK818417 \\
\hline NRRL34118 & F. oxysporum & N/A & California, U.S.A. & N/A & - & MK806586 & MK818423 \\
\hline UCR2575 & Colletotrichum gloeosporoides & Citrus reticulata & California, U.S.A. & N/A & - & MK447629 & - \\
\hline UCR7052 & Alternaria alternata & Persea americana & California, U.S.A. & N/A & - & MK101044 & - \\
\hline UCR5314 & Neofusicoccum parvum & Salix lasiolepis & California, U.S.A. & N/A & - & MH191115 & - \\
\hline UCR3250 & Clonostachys rosea & Persea americana & California, U.S.A. & N/A & - & MK101045 & - \\
\hline UCR7070 & Penicillium expansum & Persea americana & California, U.S.A. & N/A & - & MK101046 & - \\
\hline
\end{tabular}


evaluating products based on correct amplicon size. Amplicons of the five species (gBlocks; IDT) were used for generation of standard curves and positive controls for nonquantitative experiments. Lyophilized samples or the dsDNA gBlocks were suspended in nuclease free water (Thermo Fisher Scientific), and DNA concentration was quantified using a Nanodrop 2000c (Thermo Fisher Scientific). The number of copies per microliter was calculated using the following equation provided by the manufacturer:

$$
\begin{aligned}
(C) & (M)\left(1 \times 10^{-15} \mathrm{~mol} / \mathrm{fmol}\right) \text { (Avogadro's number) } \\
= & \text { copy number } / \text { microliter }
\end{aligned}
$$

where $C$ is the current concentration of the gBlocks Gene Fragment in nanograms per microliter and $M$ is the molecular weight in femtomoles per nanogram provided by the manufacturer. The gBlocks were then diluted to a concentration of $1 \times 10^{6}$ copies per $1 \mu l$ for standard curve generation, and they were diluted 10-fold to a concentration of 1 copy per microliter. Limit of detection (LoD) for each assay was determined following the MIQE guidelines (Bustin et al. 2009), which is the number of copies required for reproducible detection and quantification across experimental assays. Quantification cycle $(\mathrm{Cq})$ values were generated from CFX manager (v.3.1; Bio-Rad), with the Cq determination set to a single threshold defined as 30 relative florescence units (RFU) baseline threshold value. Singleplex reaction $\mathrm{Cq}$ values from each speciesspecific primer/probe were set to multiplex reaction $\mathrm{Cq}$ values for either PSHB or KSHB assay at a high concentration of $1 \times 10^{6}$ copies per $1 \mu \mathrm{l}$ of gBlocks and an LoD concentration of $1 \times 10^{2}$ copies per $1 \mu \mathrm{l}$ (Table 3). Intraassay and interassay $\mathrm{Cq}$ values across six experimental plates were also analyzed with the same experimental parameters mentioned above (Table 4). For PSHB assays, gBlocks dsDNA standards were mixed at a 1:1:1 ratio/reaction for each target species (F. euwallaceae/G. euwallaceae/P. pembeum) of gBlocks suspensions. For KSHB assays, gBlocks standards were mixed at a 1:1 ratio/reaction for each target species (F. kuroshium/G. kuroshium) of gBlocks suspensions. For all quantification experiments, a total of five multiplexed standards were used per assay, ranging from a high concentration of $1 \times 10^{6}$ copies per $1 \mu$ l diluted 10 -fold to the LoD of 100 copies per $1 \mu \mathrm{l}$. Multiplex assays were also tested for specificity to their target species by testing DNA extracts $(10 \mathrm{ng} / \mu \mathrm{l})$

\begin{tabular}{|c|c|c|c|c|c|}
\hline $\begin{array}{l}\text { Oligo } \\
\text { identification }\end{array}$ & Sequence $\left(5^{\prime} \rightarrow 3^{\prime}\right)$ with modifications ${ }^{b}$ & $\begin{array}{c}\text { Product } \\
\text { length (bp) }\end{array}$ & $\begin{array}{c}\text { Temperature } \\
\left({ }^{\circ} \mathrm{C}\right)\end{array}$ & GC $(\%)$ & Assay target ${ }^{\mathbf{a}}$ \\
\hline FUEU-btF & GTTACCTGACCTGCTCTGCC & 76 & 57.9 & 60 & PSHB \\
\hline FUEU-btR & ACGGCTGGGAAATGTTAGC & - & 55.6 & 52.6 & PSHB \\
\hline FUEU-bt-probe & /56-FAM/AGTTTTGTT/ZEN/TTGACATTGGTCGAGCAA/3IABkFQ/ & - & 59.9 & 37 & PSHB \\
\hline GREU-btF & GATACCTCACCTGCTCTGCT & 143 & 56.4 & 55 & PSHB \\
\hline GREU-btR & ACCACGGCTATTGTGAACAG & - & 55.2 & 50 & PSHB \\
\hline GREU-bt-probe & /5TEX615/TGCTGCTTGCTGTGCTCGATTTGTTC/3IAbRQSp/ & - & 62.1 & 50 & PSHB \\
\hline PAPE-btF & CAGTGCGTAAGTAGCCGACTC & 126 & 57.5 & 57.1 & PSHB \\
\hline PAPE-btR & GAGATGGTCTGCCAGAAAGC & - & 55.9 & 55 & PSHB \\
\hline PAPE-bt-probe & /5Cy55/ATACTGACGATGCTTCACAGGGTAACC/3IAbRQSp/ & - & 60 & 48.2 & PSHB \\
\hline FUKU-btF & GTTACCTGACCTGCTCTGCT & 78 & 56.8 & 55 & KSHB \\
\hline FUKU-btR & CCACGGCTGGGAAATATTAGT & - & 54.7 & 47.6 & KSHB \\
\hline FUKU-bt-probe & /5HEX/AGTTTTGGT/ZEN/CTGACATTTGTTGAACAA/3IABkFQ/ & - & 58.6 & 37 & KSHB \\
\hline GRKU-btF & GCTACCTCACCTGCTCTGCT & 142 & 59.2 & 60 & KSHB \\
\hline GRKU-btR & CCACGGCTATTGTGAAGAAAC & - & 54.1 & 47.6 & KSHB \\
\hline GRKU-bt-probe & /5Cy5/TGCTGCTTGTTGTGCTTGACTTGTTC/3IAbRQSp/ & - & 60.4 & 46.2 & KSHB \\
\hline
\end{tabular}

Table 2. List of the oligonucleotides used in this study

a FUEU, Fusarium euwallaceae; FUKU, Fusarium kuroshium; GREU, Graphium euwallaceae; GRKU, Graphium kuroshium; KSHB, Kuroshio shot hole borer; PAPE, Paracremonium pembeum; PSHB, polyphagous shot hole borer

${ }^{b}$ Letters in bold indicate single-nucleotide polymorphisms between the members of the same genus.

\begin{tabular}{|c|c|c|c|c|c|}
\hline $\begin{array}{l}\text { Concentration } \\
\text { (copies per microliter) }^{\mathrm{a}}\end{array}$ & Assay type $^{b}$ & Reaction type & Target species & Fluorophore & Cq values $(\mu \pm \sigma)$ \\
\hline $1,000,000$ & PSHB complex & Singleplex & Fusarium euwallaceae & FAM & $18.56 \pm 0.06$ \\
\hline $1,000,000$ & PSHB complex & Multiplex & Fusarium euwallaceae & FAM & $18.11 \pm 0.02$ \\
\hline $1,000,000$ & PSHB complex & Singleplex & Graphium euwallaceae & TEX 615 & $17.56 \pm 0.03$ \\
\hline $1,000,000$ & PSHB complex & Multiplex & Graphium euwallaceae & TEX 615 & $17.66 \pm 0.27$ \\
\hline $1,000,000$ & PSHB complex & Singleplex & Paracremonium pembeum & Cy5.5 & $18.09 \pm 0.06$ \\
\hline $1,000,000$ & PSHB complex & Multiplex & Paracremonium pembeum & Cy5.5 & $17.37 \pm 0.11$ \\
\hline $1,000,000$ & KSHB complex & Singleplex & Fusarium kuroshium & HEX & $18.86 \pm 0.46$ \\
\hline $1,000,000$ & KSHB complex & Multiplex & Fusarium kuroshium & HEX & $18.49 \pm 0.13$ \\
\hline $1,000,000$ & KSHB complex & Singleplex & Graphium kuroshium & Cy5 & $18.19 \pm 0.36$ \\
\hline $1,000,000$ & KSHB complex & Multiplex & Graphium kuroshium & Cy5 & $18.07 \pm 0.15$ \\
\hline 100 & PSHB complex & Singleplex & Fusarium euwallaceae & FAM & $31.06 \pm 0.12$ \\
\hline 100 & PSHB complex & Multiplex & Fusarium euwallaceae & FAM & $30.89 \pm 0.12$ \\
\hline 100 & PSHB complex & Singleplex & Graphium euwallaceae & TEX 615 & $30.64 \pm 0.30$ \\
\hline 100 & PSHB complex & Multiplex & Graphium euwallaceae & TEX 615 & $30.94 \pm 0.41$ \\
\hline 100 & PSHB complex & Singleplex & Paracremonium pembeum & Cy5.5 & $30.77 \pm 0.14$ \\
\hline 100 & PSHB complex & Multiplex & Paracremonium pembeum & Cy5.5 & $29.79 \pm 0.33$ \\
\hline 100 & KSHB complex & Singleplex & Fusarium kuroshium & HEX & $32.42 \pm 0.30$ \\
\hline 100 & KSHB complex & Multiplex & Fusarium kuroshium & HEX & $32.63 \pm 0.29$ \\
\hline 100 & KSHB complex & Singleplex & Graphium kuroshium & Cy5 & $32.73 \pm 0.19$ \\
\hline 100 & KSHB complex & Multiplex & Graphium kuroshium & Cy5 & $32.05 \pm 0.54$ \\
\hline
\end{tabular}

Table 3. Comparison of $(\mathrm{Cq})$ values from each target species in singleplex and multiplex reactions

${ }^{a}$ Concentrations derived from diluted gBlocks suspension.

${ }^{\mathrm{b}}$ KSHB, Kuroshio shot hole borer; PSHB, polyphagous shot hole borer. 
from nontarget species (Table 1) while using DNA extracts from type isolates as positive controls individually as well as a mixed reaction of type isolates (Table 1).

Detection from inoculated wood with target fungal species. Avocado stems from 2-year old cultivar Zutano seedlings were used for artificial inoculation of the target fungal species: F. euwallaceae, $F$. kuroshium, G. euwallaceae, G. kuroshium, and P. pembeum. The cuttings (20-cm long) were surface sterilized by soaking in a $10 \%$ household bleach solution for $10 \mathrm{~min}$; then, they were rinsed with deionized water and allowed to dry. Although the use of a fungal plug of colonized agar is reported in other studies (Lynch et al. 2016; Pouzoulet et al. 2013), we decided to mimic the inoculation of these fungi by the vector by drilling small holes and depositing individual as well as mixed spore suspensions in combinations that exist in the field. In addition, two mixed inoculations not found in the field were also included (Fusarium spp. mix and Graphium spp. mix). Spore suspensions were prepared from isolates of $F$. euwallaceae, $F$. kuroshium, G. euwallaceae, G. kuroshium, and P. pembeum (Tables 5 and 6) by scraping 1-week-old sporulating cultures grown on PDA with a sterile inoculating loop. These were streaked onto fresh PDA media plates amended with $0.01 \%$ tetracycline hydrochloride and grown at $25^{\circ} \mathrm{C}$ for 3 days. Five milliliters of sterile distilled water was added to each plate and agitated with a sterile bent glass rod to release the spores into suspension before passing through a layer of

Table 4. Evidence of reproducibility across experimental plates

\begin{tabular}{|c|c|c|c|c|c|c|c|}
\hline $\begin{array}{l}\text { Concentration } \\
\text { (copies per } \\
\text { microliter) }\end{array}$ & Assay type ${ }^{b}$ & Target species & Fluorophore & Cq values $(\mu \pm \sigma)^{c}$ & $\mathrm{CV}(\%)$ & Cq values $(\mu \pm \sigma)^{d}$ & CV $(\%)$ \\
\hline $1,000,000$ & PSHB complex & Fusarium euwallaceae & FAM & $18.00 \pm 0.33$ & 1.86 & $18.07 \pm 0.30$ & 1.66 \\
\hline 100,000 & PSHB complex & F. euwallaceae & FAM & $21.10 \pm 0.27$ & 1.36 & $21.19 \pm 0.19$ & 0.91 \\
\hline 10,000 & PSHB complex & F. euwallaceae & FAM & $24.44 \pm 0.14$ & 0.57 & $24.53 \pm 0.20$ & 0.82 \\
\hline 1,000 & PSHB complex & F. euwallaceae & FAM & $27.94 \pm 0.14$ & 0.51 & $27.98 \pm 0.33$ & 1.19 \\
\hline 100 & PSHB complex & F. euwallaceae & FAM & $30.54 \pm 0.03$ & 0.09 & $30.75 \pm 0.36$ & 1.19 \\
\hline $1,000,000$ & PSHB complex & Graphium euwallaceae & TEX 615 & $17.75 \pm 0.29$ & 1.66 & $17.41 \pm 0.39$ & 2.24 \\
\hline 100,000 & PSHB complex & G. euwallaceae & TEX 615 & $20.57 \pm 0.21$ & 1.04 & $20.42 \pm 0.32$ & 1.57 \\
\hline 10,000 & PSHB complex & G. euwallaceae & TEX 615 & $23.80 \pm 0.34$ & 1.43 & $23.73 \pm 0.35$ & 1.47 \\
\hline 1,000 & PSHB complex & G. euwallaceae & TEX 615 & $27.20 \pm 0.43$ & 1.59 & $27.01 \pm 0.45$ & 1.65 \\
\hline 100 & PSHB complex & G. euwallaceae & TEX 615 & $30.05 \pm 0.43$ & 1.44 & $30.02 \pm 0.37$ & 1.23 \\
\hline $1,000,000$ & PSHB complex & Paracremonium pembeum & Су5.5 & $17.40 \pm 0.23$ & 1.30 & $17.49 \pm 0.29$ & 1.68 \\
\hline 100,000 & PSHB complex & P. pembeum & Cy5.5 & $20.68 \pm 0.20$ & 0.98 & $20.68 \pm 0.38$ & 1.82 \\
\hline 10,000 & PSHB complex & P. pembeum & Cy5.5 & $24.17 \pm 0.14$ & 0.57 & $24.10 \pm 0.30$ & 1.25 \\
\hline 1,000 & PSHB complex & P. pembeum & Cy5.5 & $27.71 \pm 0.54$ & 1.95 & $27.55 \pm 0.39$ & 1.43 \\
\hline 100 & PSHB complex & P. pembeum & Cy5.5 & $30.70 \pm 0.37$ & 1.23 & $30.76 \pm 0.28$ & 0.92 \\
\hline $1,000,000$ & KSHB complex & F. kuroshium & HEX & $18.38 \pm 0.15$ & 0.83 & $18.41 \pm 0.20$ & 1.09 \\
\hline 100,000 & KSHB complex & F. kuroshium & HEX & $22.18 \pm 0.21$ & 1.93 & $21.9 \pm 0.49$ & 2.26 \\
\hline 10,000 & KSHB complex & F. kuroshium & HEX & $25.50 \pm 0.32$ & 1.27 & $25.39 \pm 0.27$ & 1.04 \\
\hline 1,000 & KSHB complex & F. kuroshium & HEX & $28.95 \pm 0.69$ & 2.38 & $29.19 \pm 0.57$ & 1.96 \\
\hline 100 & KSHB complex & F. kuroshium & HEX & $32.18 \pm 0.22$ & 0.68 & $32.72 \pm 0.50$ & 1.54 \\
\hline $1,000,000$ & KSHB complex & G. kuroshium & Cy5 & $18.18 \pm 0.17$ & 0.95 & $18.14 \pm 0.21$ & 1.16 \\
\hline 100,000 & KSHB complex & G. kuroshium & Cy5 & $21.44 \pm 0.35$ & 0.35 & $21.36 \pm 0.53$ & 2.50 \\
\hline 10,000 & KSHB complex & G. kuroshium & Cy5 & $24.80 \pm 0.14$ & 0.55 & $24.7 \pm 0.34$ & 1.38 \\
\hline 1,000 & KSHB complex & G. kuroshium & Cy5 & $28.43 \pm 0.57$ & 1.99 & $28.48 \pm 0.47$ & 1.64 \\
\hline 100 & KSHB complex & G. kuroshium & Cy5 & $31.63 \pm 0.88$ & 2.81 & $31.95 \pm 0.94$ & 2.95 \\
\hline
\end{tabular}

${ }^{a}$ Concentrations derived from diluted gBlocks suspensions.

${ }^{\mathrm{b}} \mathrm{CV}$, coefficient of variation; KSHB, Kuroshio shot hole borer; PSHB, polyphagous shot hole borer.

${ }^{c}$ Intraassay quantification cycle $(\mathrm{Cq})$ values were generated across one experimental plate $(N=3)$.

d Interassay $\mathrm{Cq}$ values were generated across six experimental plates $(N=18)$.

Table 5. Comparison of recovery and quantification of fungi from detached shoots inoculated with shot hole borer (SHB) fungi

\begin{tabular}{lccc}
\hline Treatment $^{\mathbf{a}}$ & SHB complex $^{\mathbf{b}}$ & \% Recovery (isolation) $^{\mathbf{c}}$ & \% Recovery (qPCR) $^{\mathbf{d}}$ \\
\hline Fusarium euwallaceae & PSHB & 80 & 100 \\
F. euwallaceae/Graphium euwallaceae & PSHB & $40 / 20$ & $100 / 30$ \\
F. euwallaceae/Paracremonium pembeum & PSHB & $60 / 0$ & $80 / 0$ \\
F. euwallaceae/G. euwallaceae/P. pembeum & PSHB & $50 / 0 / 0$ & $100 / 20 / 0$ \\
G. euwallaceae & PSHB & 0 & 60 \\
G. euwallaceae/P. pembeum & PSHB & $10 / 10$ & $80 / 0$ \\
P. pembeum & PSHB & 0 & 90 \\
F. kuroshium & KSHB & 70 & 100 \\
F. kuroshium/G. kuroshium & KSHB & $70 / 10$ & $70 / 20$ \\
G. kuroshium & KSHB & 0 & 50 \\
F. euwallaceae/F. kuroshium & N/A & 70 & $100 / 100$ \\
G. euwallaceae/G. kuroshium & N/A & $0 / 0$ & $80 / 80$ \\
\hline
\end{tabular}

a All inoculations were done on detached shoots $(N=10)$ by depositing 1,000 spores of each species into artificial wounds.

$\mathrm{b}$ Mixed inoculations of species from both complexes have not been observed in nature. Therefore, they are represented by N/A. KSHB, Kuroshio shot hole borer; PSHB, polyphagous shot hole borer.

${ }^{\mathrm{c}}$ All isolations were done on potato dextrose agar amended with $0.01 \%$ tetracycline hydrochloride.

${ }^{\mathrm{d}}$ Recovery values are from quantitative results. qPCR, quantitative PCR. 
sterile Kimwipe to remove large mycelial fragments. All spore suspensions were quantified using a Bright-Line hemocytometer (Hausser Scientific) and diluted to $1 \times 10^{6}$ spores per $1 \mathrm{ml}$. To inoculate shoots, five wounds were made per shoot with two replicate shoots per fungal species using a rotary tool (Dremel 7700 Series) with a flamed 7114 diamond wheel point attachment (Dremel). Ten microliters of a spore suspension and mixed combination treatments (Tables 5 and 6) were pipetted in the wounds and wrapped with parafilm for a total of 13 treatments, including a sterile watertreated control. These were placed in plastic containers lined with paper towels soaked with deionized water to maintain humidity. Shoots were destructively sampled after 4 weeks, and $1 \times 1$-cm wood pieces were sampled from the discolored margins. These were plated on PDA amended with $0.01 \%$ tetracycline hydrochloride to recover the inoculated fungi. The remaining discolored wood $(\sim 200 \mathrm{mg})$ was weighed and frozen at $-80^{\circ} \mathrm{C}$. DNA extracts from the wood were used in quantification experiments for both PSHB and KSHB assays in triplicate, and DNA concentrations were normalized to $10 \mathrm{ng}$ per reaction. Fungi were isolated on PDA amended with $0.01 \%$ tetracycline to compare recovery from conventional isolation methods (Table 5). Controls for the experimental plates included a negative control (extract from water-treated wood) and an NTC. The SPUD test (Nolan et al. 2006) was also performed to verify that the noninoculated plant extract did not inhibit the PCR reaction significantly by spiking F. euwallaceae, F. kuroshium, G. euwallaceae, G. kuroshium, and $P$. pembeum isolate DNA templates at $10 \mathrm{ng}$ per reaction with an equal concentration of avocado DNA extract.

Quantification of fungal symbionts from the beetle mycangia. Ten adult female PSHB and KSHB beetles were obtained from laboratory-reared sawdust media colonies maintained with methods similar to those in the work by Cooperband et al. (2016). For quantification experiments, DNA extracts from beetle heads were normalized to $10 \mathrm{ng}$ per reaction and tested in triplicate in both PSHB and KSHB assays.

Field sampling. Locations in Riverside (6), Orange (15), and San Diego (5) Counties were surveyed for shot hole borer (SHB) attacks, and samples were collected from Platanus racemosa (278), Populus spp. (59), and Salix spp. (11) if visible symptoms of frass, sapping, and/or gumming were present with visible entry holes as described in Eskalen et al. (2013). These trees were chosen, because these hosts are favored by this pest and display obvious combinations of the symptoms described above. Trees exhibiting symptoms were sampled in five different areas per tree with visible SHB entry holes, and samples were collected by cutting an $\sim 1 \times 1 \times 1$-cm cube around the entry hole with a clean Buck knife (Buckknife) to remove the infested tissue as well as the beetle if present. The knife was cleaned with $95 \%$ ethanol via spray bottle after each sample. After samples were collected, they were deposited in $1.5-\mathrm{ml}$ microcentrifuge tubes, labeled, placed in a cooler, and then, transported back to the laboratory (California Department of Food and Agriculture [CDFA] plant pest permit \#2887) for storage at $4{ }^{\circ} \mathrm{C}$ until processing. Wood samples were surface sterilized by dipping the wood sample in $95 \%$ ethanol and flamed; then, discolored margins were dissected with sterile tools near the entry holes and plated on PDA amended with $0.01 \%$ tetracycline hydrochloride. The remaining sample was placed in a sterile $1.5-\mathrm{ml}$ microcentrifuge tube and frozen at $-80^{\circ} \mathrm{C}$. The isolation plates were incubated at $28^{\circ} \mathrm{C}$ for at least 3 days. The fungal colonies used in this trial were selected based on their morphology as described by Freeman et al. (2013), Lynch et al. (2016), and Na et al. (2018). Fungal colonies of interest were sampled with a sterile toothpick and placed in a $0.5-\mathrm{ml}$ microfuge tube with $25 \mu \mathrm{l}$ of sterile water to make a spore suspension. Resultant spore suspensions were used as a template for both PSHB and KSHB assays for downstream qualitative identification. GPS locations of all trees as well as sample information were recorded using custom-built forms on the iForm application (Zurion Software Inc.). All maps were created using $\mathrm{R}$ (version 3.4.3) (RC Team 2013) and the "leaflet" package (Cheng et al. 2017).

Statistical analyses. All statistical analyses were performed using $\mathrm{R}$ (version 3.4.3) (RC Team 2013). Analyses of $\mathrm{Cq}$ values from singleplex/multiplex and intraassay/interassay comparisons were done using the "plyr" package (Wickham 2011) to calculate means, standard deviation, standard error, and coefficient of variation values. Quantitative experiments were analyzed using estimated starting quantities (SQs) across all treatments with the data separated by target species. SQs from the samples were calculated from a standard curve from each experimental plate run. Regression analysis using log-transformed SQ values from inoculation treatments was performed by target species (fluorophore) (Table 6). Because of the overdispersion of the data as the variance exceeds the mean, which was confirmed using the dispersiontest function from the package "AER" (Kleiber et al. 2015), regression analysis using a generalized linear model with a negative binomial link function was performed using the "MASS" package (Venables and Ripley 2002). Detached shoot inoculation post hoc analysis was done by performing an all pairwise Tukey's test for the comparison across all responses to treatments by target. PSHB/KSHB female heads post hoc analysis was done by constructing a contrast matrix to compare all responses to target species by biological sample (PSHB or KSHB) of interest. Then, the "multcomp" package (Hothorn et al. 2008) was used with link function glht for comparison of all target species to each other based on the matrix. All bar plots were generated using "ggplot2"

Table 6. Quantification of fungi from detached shoots inoculated with shot hole borer (SHB) fungi

\begin{tabular}{|c|c|c|c|c|c|c|c|}
\hline \multirow[b]{2}{*}{ Treatment $^{\mathbf{a}}$} & \multirow[b]{2}{*}{ SHB complex ${ }^{b}$} & \multirow{2}{*}{$\begin{array}{l}\text { Estimated starting } \\
\text { quantity }(\mu \pm \sigma)^{\mathrm{c}}\end{array}$} & \multicolumn{5}{|c|}{$P$ value $^{\mathrm{d}}$} \\
\hline & & & FAM & TEX 615 & Cy5.5 & HEX & Cy5 \\
\hline Fusarium euwallaceae & PSHB & $596.8 \pm 213.5$ & Reference & - & - & - & - \\
\hline F. euwallaceae/Graphium euwallaceae & PSHB & $470.9 \pm 304.47 / 352.4 \pm 221.2$ & 0.634 & 0.002 & - & - & - \\
\hline F. euwallaceae/Paracremonium pembeum & PSHB & $1,242.6 \pm 607 / \mathrm{ND}$ & 0.172 & - & ND & - & - \\
\hline F. euwallaceae/G. euwallaceae/P. pembeum & PSHB & $876.2 \pm 235 / 137.9 \pm 17.2 / \mathrm{ND}$ & 0.439 & 0.462 & ND & - & - \\
\hline G. euwallaceae & PSHB & $108.51 \pm 12.3$ & - & Reference & - & - & - \\
\hline G. euwallaceae/P. pembeum & PSHB & $131.4 \pm 15.7 / \mathrm{ND}$ & - & 0.387 & ND & - & - \\
\hline P. pembeum & PSHB & $119.1 \pm 25.6$ & - & - & Reference & - & - \\
\hline F. kuroshium & KSHB & $2,287.5 \pm 534.2$ & - & - & - & Reference & - \\
\hline F. kuroshium/G. kuroshium & KSHB & $1,260.3 \pm 667.9 / 152.4 \pm 28.4$ & - & - & - & $<0.001$ & 0.887 \\
\hline G. kuroshium & KSHB & $170.4 \pm 55$ & - & - & - & - & Reference \\
\hline F. euwallaceae/F. kuroshium & N/A & $1,593.6 \pm 594.5 / 3,355 \pm 1,086.9$ & 0.048 & - & & 0.700 & - \\
\hline G. euwallaceae/G. kuroshium & N/A & $171 \pm 93.3 / 344.5 \pm 174.8$ & - & 0.012 & - & - & 0.167 \\
\hline
\end{tabular}

a All inoculations were done on detached shoots $(N=10)$ by depositing 1,000 spores of each species into artificial wounds.

${ }^{\mathrm{b}}$ Mixed inoculations of species from both complexes have not been observed in nature; therefore, they are represented with N/A. KSHB, Kuroshio shot hole borer; PSHB, polyphagous shot hole borer.

${ }^{\mathrm{c}}$ Copies per microliter. ND, not detected.

${ }^{\mathrm{d}} \mathrm{P}$ values are the results of a generalized linear model with negative binomial link function using the target response inoculated alone as the reference. Bold values are significant $(P<0.05)$ while dashes indicate the target was not included in the treatment. 
(Wickham 2016). Statistics for the field survey recovery frequencies were also done using logistic regression measuring the frequency of recovery by plant species and recovered fungus genera using the binomial link function.

\section{Results}

Primer/probe design and validation. Development of the species-specific primers for fungi associated with PSHB and KSHB was based on the nucleotide sequence alignment of the TUB2 gene sequences, which were found to contain one copy per haploid genome by using the FASTA program (Pearson 2016) against available AFC accessions. ITS and TEF1 genes had few polymorphic regions to exploit within targeted genera and the species that we targeted. In silico results from the FASTA program indicated that target Fusarium spp. have $<100 \%$ matches with primer sets and probes to available AFC genome accessions. BLAST indicated that most specific primer sets and probes had $<100 \%$ matches to existing accessions other than the targets, except for the $F$. euwallaceae reverse primer, which had a $100 \%$ match to a closely related species within the Fusarium solani species complex (FSSC); however, the probes were all found to be specific, with $<100 \%$ matches to existing accessions other than the targets. It should be noted that Graphium spp. and Paracremonium spp. have limited TUB2 accessions in GenBank. Preliminary primer optimization using conventional PCR showed that the optimum annealing/extension temperature for all specific primer sets of interest was $60^{\circ} \mathrm{C}$, which amplified products of expected sizes listed in Table 2. No PCR products were observed when nontarget DNA extracts (Table 1) were tested with species-specific primer sets.

Development of multiplex real-time PCR assays. Initially, we intended to develop a multiplex assay that would allow for the detection of all fungi associated with PSHB and KSHB simultaneously in one reaction; however, we had issues with significant differences in detection (Cq values) using DNA from all five targets present in multiplex versus singleplex reactions (data not shown). Therefore, we decided to develop two assays: a PSHB assay and a KSHB assay; they did not show significantly higher $\mathrm{Cq}$ values $(P>0.05)$ in multiplex assays compared with singleplex assays (Table 3). Interassay and intraassay $\mathrm{Cq}$ values were not significantly different across the experimental plates tested (Table 4). NTC values for target Fusarium spp. produced a signal after 33 cycles; therefore, cycling conditions should not go for $>33$ cycles. Reaction efficiencies ranged from 88.2 to $104.3 \%$, with $R^{2} \geq 0.992$. These were obtained across all targets based on the slopes generated from standard curves of synthesized dsDNA suspensions (gBlocks), covering a dynamic range of $1 \times$ $10^{6}$ to $1 \times 10^{2}$ copies per $1 \mu l$ for each target species (Fig. 1 ). The LoD was determined to be $1 \times 10^{2}$ copies per $1 \mu$, because concentrations below this value did not reproducibly produce a detectable signal. Similar efficiencies and LoDs were found for singleplex conditions compared with multiplex conditions.

Detection from artificially inoculated wood. Among PSHBassociated fungi, F. euwallaceae was detected by the qPCR assay in 80 to $100 \%$ of individual and mixed fungal treatments that were inoculated with this species, whereas it was recovered 40 to $80 \%$ of the time from conventional isolation methods depending on the inoculum (Table 5). G. euwallaceae had a higher detection rate from qPCR (30 to 80\%) depending on the inoculation combination, which was higher than conventional isolation (0 to 20\%) (Table 5). P. pembeum was detected in $90 \%$ of treatments from qPCR assays from nonmixed treatments and only $10 \%$ from a mixed inoculation of $G$. euwallaceae and $P$. pembeum, whereas the other treatments with this fungus were not detected by qPCR or conventional isolation (Table 5). For fungi associated with KSHB, F. kuroshium was detected by the qPCR assay from 70 to $100 \%$ of individual and mixed fungal inoculations and $70 \%$ by conventional isolation. Detection of G. kuroshium from qPCR was 20 to $50 \%$ for individual and mixed inoculations compared with 0 to $10 \%$ from conventional methods (Table 5). Inoculations simulating a mix of fungi from both PSHB and KSHB resulted in $100 \%$ detection of $F$. euwallaceae and $F$. kuroshium from the respective qPCR assays and 70\% of Fusarium spp. from conventional isolation (Table 5). It should be noted that $F$. euwallaceae and $F$. kuroshium cannot be differentiated when recovered from conventional isolation. G. euwallaceae and G. kuroshium both resulted in detection of $80 \%$ from the qPCR assays, but they were not recovered from conventional isolation (Table 5).

User-defined contrasts resulted in significantly greater SQs $(P<$ 0.05 ) of $G$. euwallaceae (TEX 615) from a mixed inoculation of $F$. euwallaceae (FAM) and G. euwallaceae compared with G. euwallaceae inoculated alone. F. kuroshium (HEX) SQ was significantly lower $(P<0.05)$ from a mixed inoculation of $F$. kuroshium and $G$. kuroshium (CY5.5) compared with $F$. kuroshium inoculated alone. From mixed inoculations of fungi from both PSHB and KSHB, $F$. euwallaceae was found to have a significantly higher SQ $(P<$ 0.05 ) in a mixed treatment of $F$. euwallaceae and $F$. kuroshium compared with $F$. euwallaceae inoculated alone. F. kuroshium showed no significant differences in SQ $(P>0.05)$ in the same assay. $G$. euwallaceae was found to have a significantly greater SQ $(P<$ 0.05 ) in a mixed treatment of G. euwallaceae and G. kuroshium compared with G. euwallaceae inoculated alone, whereas G. kuroshium showed no significant differences in SQ $(P>0.05)$ in the same assay (Table 6).

Quantification from beetle mycangia. Only $F$. euwallaceae and G. euwallaceae from PSHB heads and only F. kuroshium and G. kuroshium from KSHB heads (Fig. 2) were positively detected with the two qPCR assays. The SQ of $F$. euwallaceae in PSHB heads was found to be significantly greater $(P<0.05)$ than that of $G$. euwallaceae. In the KSHB heads, the SQ of $F$. kuroshium was not found to be significantly greater $(P>0.05)$ than that of G. kuroshium. However, $F$. euwallaceae from PSHB had a significantly greater SQ than $F$. kuroshium in KSHB.

Recovery from field samples. Samples recovered from $65.4 \%$ of the locations sampled were positive for signs and symptoms of FD, and this was confirmed by detecting fungi associated with the pests through conventional isolation methods. DNA extracted from wood samples failed to produce detectable signals for both assays and failed the SPUD test (Nolan et al. 2006) when positive controls were spiked with DNA extract from field samples. Because of the lack of PCR amplification of DNA from field samples, conventional isolation from wood was performed with downstream identification of recovered fungi by qPCR. Recovery rates from the three different plant species sampled (Platanus racemosa, Populus spp., and Salix spp.) were $88.3,72.7$, and $56.1 \%$, respectively, for Fusarium spp. and 11.5 , 0, and $21.1 \%$, respectively, for Graphium spp. Samples positive for target Fusarium spp. were recovered significantly more than $(P<0.001)$ Graphium spp. from wood in all three plant species sampled (Fig. 3). Samples positive for Fusarium spp. were recovered significantly less frequently $(P<0.001)$ in Salix spp. compared with Platanus racemosa, whereas Graphium spp. were recovered more in Salix spp. $(P=0.075)$ compared with Platanus racemosa (Fig. 3). Isolates of Fusarium spp. and Graphium spp. from both beetles were morphologically indistinguishable; therefore, downstream identification of the fungal cultures was through using both PSHB and KSHB qPCR assays. Of the surveyed counties (Fig. 4), in Orange County, both complexes were present in close proximity, mainly in Lake Forest and Mission Viejo. On five trees in a 4-mile radius within this region, both fungi associated with PSHB and KSHB were confirmed from the same tree. Both PSHB and KSHB were confirmed to be present in both Orange and San Diego Counties, and areas sampled in West Riverside County were all negative, despite some trees exhibiting an entry hole; however, PSHB has been found in northern parts of Riverside County in proximity to the Santa Ana River previous to this study (A. Eskalen, unpublished data).

\section{Discussion}

Identification of PSHB and KSHB attacks can be difficult, because the beetles are often absent from areas of visible attack and commonly mistaken for other benign ambrosia or bark beetles. In our study, a real-time PCR assay was developed to address this issue and provide a rapid and reliable identification method to target specific fungi associated with PSHB and KSHB in California that is not dependent on the beetle being present in samples. Polymorphic 
regions of the $T U B 2$ were exploited to design, optimize, and validate two novel multiplexed qPCR assays that can detect and quantitate the symbiotic fungi associated with PSHB and KSHB (Fig. 1 and Supplementary Fig. S1). The assays were shown to be sensitive, with an LoD of $1 \times 10^{2}$ copies per $1 \mu \mathrm{l}$ for all targets (Table 4), and they were able to detect targets across various sample types, with Fusarium spp. shown to be the most frequent genus detected (Figs. 2 and 3 and Tables 5 and 6). However, DNA extraction from field samples of Platanus racemosa, Populus spp., and Salix spp. failed the SPUD test (Nolan et al. 2006), most likely because of PCR inhibitors in the wood. Therefore, it is best to identify SHB attacks by isolating fungi from the wood and then, using spores from recovered fungi as a template for the multiplexed qPCR assays. These real-time molecular tools are useful to diagnose and distinguish the main symbionts of PSHB and KSHB from affected hosts in California as well as to elucidate the distribution and movement of these pests as shown from our field surveys (Fig. 4).

For this study, we chose to exploit $T U B 2$ for our assays, because it occurs as a single-copy gene in the genomes of most fungi. Selection of a region with one copy per genome can reduce the assay sensitivity, but it also reduces quantification bias (Brancart et al. 2005) and allows conversion of copy numbers to individual spores. This increases the assay utility, because spores are abundant in female SHB mycangia (Freeman et al. 2016) and fungal galleries from infested wood samples (Lynch
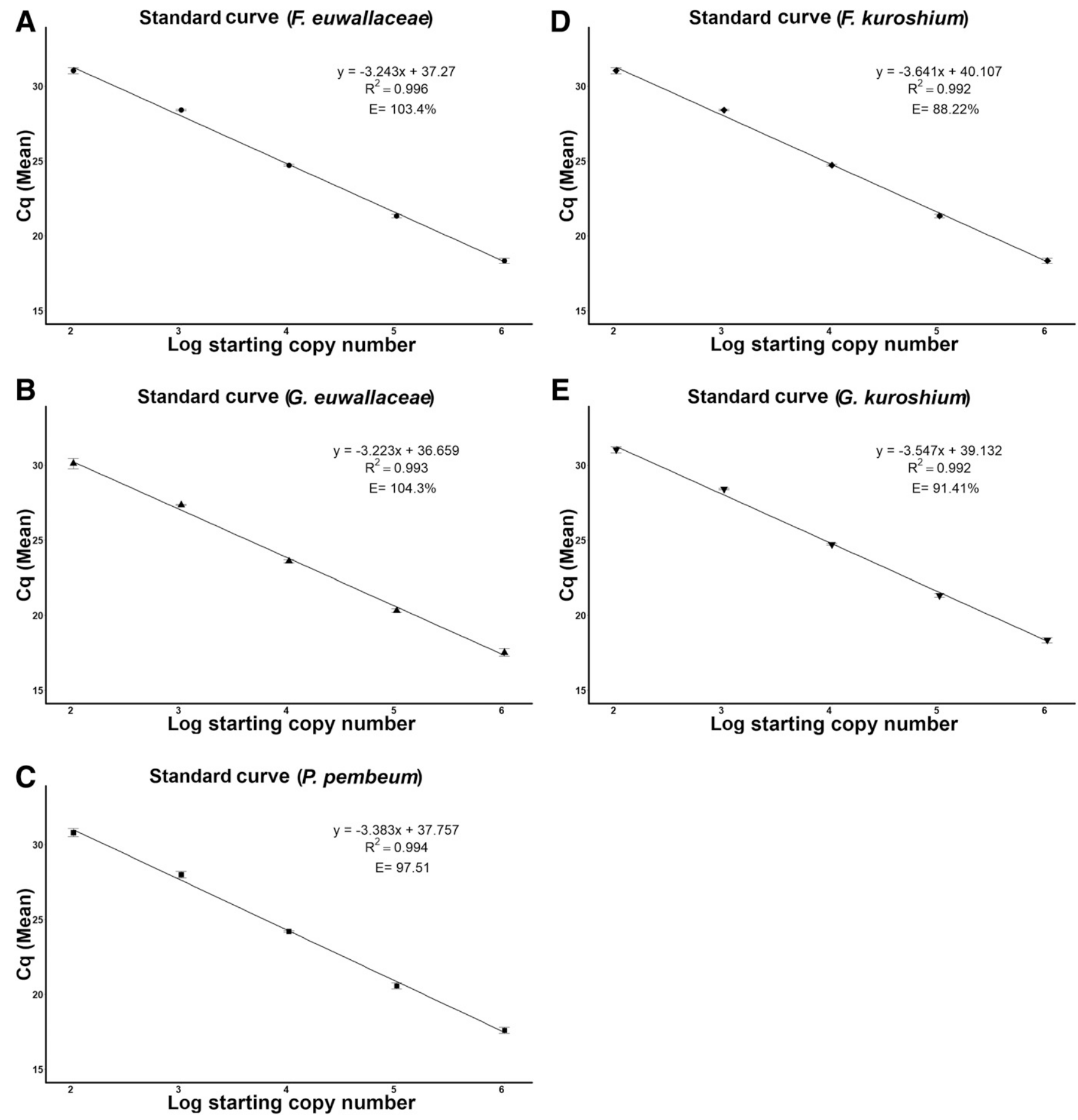

Fig. 1. Standard curves for quantitative PCR using species-specific primers to detect fungi from $A, B$, and $C$, polyphagous shot hole borer and $D$ and $E$, Kuroshio shot hole borer. Standard curves were constructed using 10-fold dilutions of synthesized dsDNA gBlocks fragments for each target from a starting quantity of $1 \times 10^{6}$ to $1 \times 10^{2}$ copies per reaction. Each reaction was done in triplicate; efficiencies were $>88.2$, and coefficient of determination was $>0.992$. A, Fusarium euwallaceae, B, Graphium euwallaceae, C, Paracremonium pembeum, D, Fusarium kuroshium, and E, Graphium kuroshium. $\mathrm{Cq}=$ quantification cycle. 
et al. 2016). The primers and hydrolysis probes selected were validated by agarose gel electrophoresis (AGE) based on expected amplicon size along with specificity testing against members of the $F$. solani and $F$. oxysporum species complexes (Table 1), because these are commonly recovered from SHB field samples from Persea americana, Platanus racemosa, and Salix spp. (A. Eskalen, unpublished data). Primers and probes were also tested against other fungal species commonly recovered from xylem tissue of these hosts, including A. alternata, $C$. rosea, N. parvum, and Penecillium digitatum, which confirmed that nonspecific amplification did not occur from these fungi.

Multiplexed assays have been shown to be effective in detecting multiple plant pathogens from wood samples using real-time methods (Li et al. 2006, 2009; Pouzoulet et al. 2013; Zitnick-Anderson et al. 2018) as well as detecting AFC fusaria associated with Euwallacea spp. from DNA extracts of fungi and beetle heads using conventional PCR methods (Short et al. 2017). The difference from the latter is that the assays presented here detect all described fungal symbionts associated with PSHB and KSHB in California by not just targeting the AFC fusaria. In addition, detection of fungi from single beetle heads was successful with these assays, where pooled samples were the only sample types to show detection from the previously mentioned conventional multiplex PCR methods targeting AFC fusaria. Although AFC fusaria were recovered most frequently from various SHB samples in previous studies (Freeman et al. 2013, 2016; Lynch et al. 2016; Mendel et al. 2012; Na et al. 2018; O'Donnell et al. 2015) as well as in this study, detecting other fungi involved in these invasive pest disease complexes can increase identification success in samples from hosts, such as Salix spp., which were shown to have greater abundance of Graphium spp. compared with other hosts tested. In previous studies, $P$. pembeum $\mathrm{CFU}$ were found to be more abundant than F. euwallaceae in gallery samples from wood in Acer negundo and Ricinus communis, whereas overall, G. euwallaceae and $P$. pembeum were more abundant in galleries compared with beetle heads (Lynch et al. 2016). Because of the possibility of higher abundance of fungal symbionts other than AFC fusaria, such as Graphium spp. and P. pembeum, in SHB samples from certain hosts, a system able to detect all known fungi associated with Euwallacea spp. would be more reliable for the identification of these pest disease complexes in invaded areas, like California.

Quantification of pathogen loads from DNA using multiplex assays has been done for plant pathogens from nonlignified (Li et al. 2009) as well as lignified tissue (Pouzoulet et al. 2013), but to our

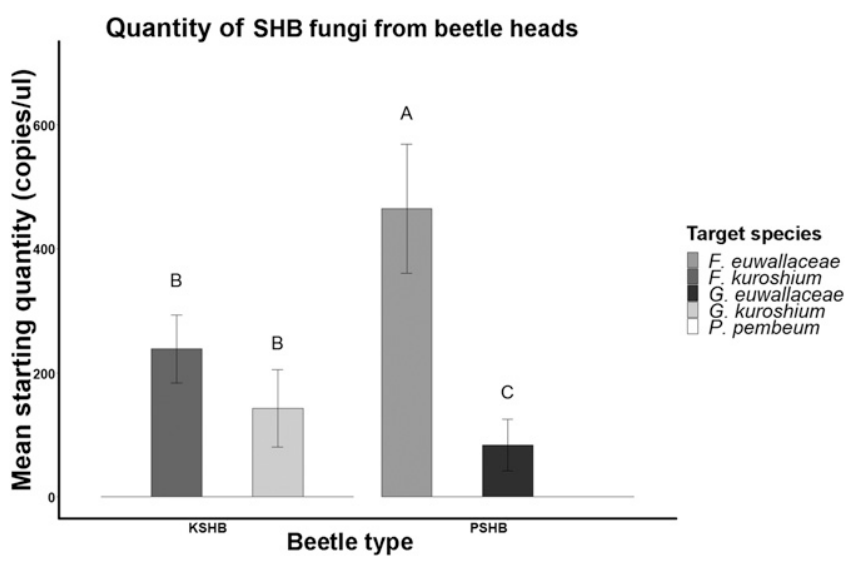

Fig. 2. Mean starting quantity of fungal symbionts recovered from DNA extracts of female Kuroshio shot hole borer (KSHB) and polyphagous shot hole borer (PSHB) heads using the multiplex real-time assays. Estimation of starting quantity was generated based on multiplexed standard curves performed on each experimental plate using synthetic dsDNA gBlocks from each target species from $1 \times 10^{6}$ to $1 \times$ $10^{2}$ copies per reaction. Vertical lines represent standard error of the mean, and $P$ values were generated using negative binomial regression analysis and post hoc analysis from user-defined contrasts using the R package "multcomp." Fusarium euwallaceae, Fusarium kuroshium, Graphium euwallaceae, Graphium kuroshium, and Paracremonium pembeum are shown. SHB, shot hole borer. knowledge, it has not been applied to an SHB-FD system. The dynamics and function of the fungi associated with Euwallacea spp. have been discussed for AFC fusaria (Eskalen et al. 2013; Freeman et al. 2013, 2016; Kasson et al. 2013; Lynch et al. 2016; Mendel et al. 2012; Na et al. 2018; O'Donnell et al. 2015), but they have been discussed less so for the function of Graphium spp. and P. pembeum in this system (Freeman et al. 2016; Lynch et al. 2016). Detection of all SHB-FD fungi with the multiplex assays using DNA extracts from artificially inoculated avocado shoots and female beetle heads had varying recovery rates (Fig. 2 and Tables 5 and 6), but it presents a platform to detect all fungi present in the PSHB and KSHB samples. The PSHB and KSHB assays can be utilized to compare relative quantity of SHB fungi within amplifiable samples to investigate effects of pesticide candidates on the quantity of these fungi from controlled in vitro and field experiments, possibly elucidating pesticides that disrupt the system for management of this invasive pest in California.

Based on field samples collected from Orange, Riverside, and San Diego Counties (Fig. 4), after identification using the multiplex assays, both PSHB and KSHB were present in northern San Diego County (Carlsbad) as well as the central east side of Orange County (Irvine/Lake Forest), where five trees were found with fungi from both beetles within these areas. In previous studies, it has been reported that ambrosia beetles can switch or steal fungal symbionts, known as promiscuous symbiosis or fungal sharing (Carrillo et al. 2014; Hulcr and Cognato 2010; Kostovcik et al. 2015); however, it is currently unknown if this phenomenon is occurring in areas, such as Orange County, where trees are supporting both PSHB and KSHB. Some invasive Euwallacea spp. have been reported to vector multiple AFC fusaria in Florida and Sri Lanka (O'Donnell et al. 2015). This raises concern for the situation in Orange County if PSHB and KSHB can accumulate new symbionts from each other, not limited to AFC fusaria, and potentially increase their arsenal to colonize woody hosts. Assessing which fungi are causing the damage becomes difficult, because PSHB fungi were found to have significant differences in pathogenicity (Lynch et al. 2016; Na et al. 2018). Molecular tools, such as the assays presented here, have potential in assessing the dynamics of these fungi among the wide range of reproductive hosts, but the PCR inhibition in wood must be overcome first. Varying conditions, such as age of gallery, host responses, and extreme tissue discoloration, may affect the samples and contribute to the PCR inhibition that has been reported in other systems (Demeke et al. 2009; Schaad and Frederick 2002). However, our diagnostic assay is still useful for qPCR identification of fungi recovered across a range of hosts (Figs. 2, 3 , and 4 and Tables 5 and 6 ) as well as for the detection of the pest in the early stages of infestation to aid in management decisions while reducing false identification.

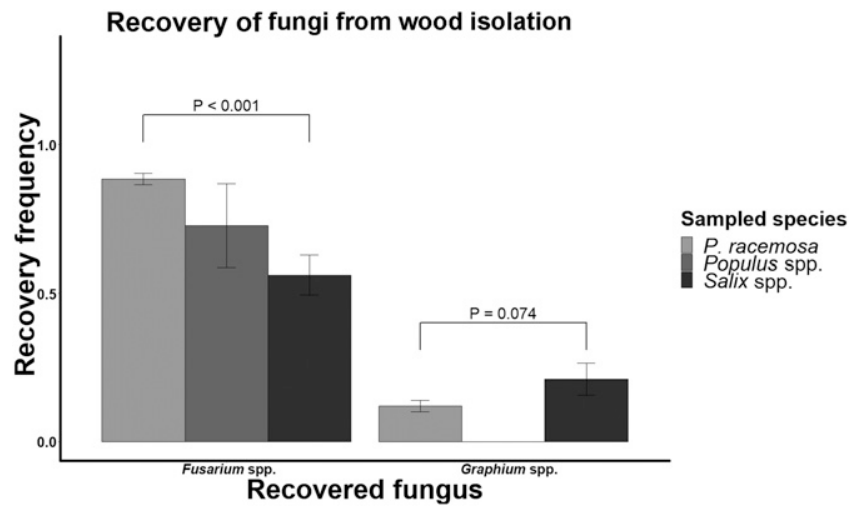

Fig. 3. Recovery frequency of Fusarium spp. and Graphium spp. from Platanus racemosa, Populus spp., and Salix spp. using conventional fungal isolation methods on potato dextrose agar amended with $0.01 \%$ tetracycline. Vertical lines represent standard error of the mean, and $\mathrm{P}$ values were generated using logistic regression analysis. 


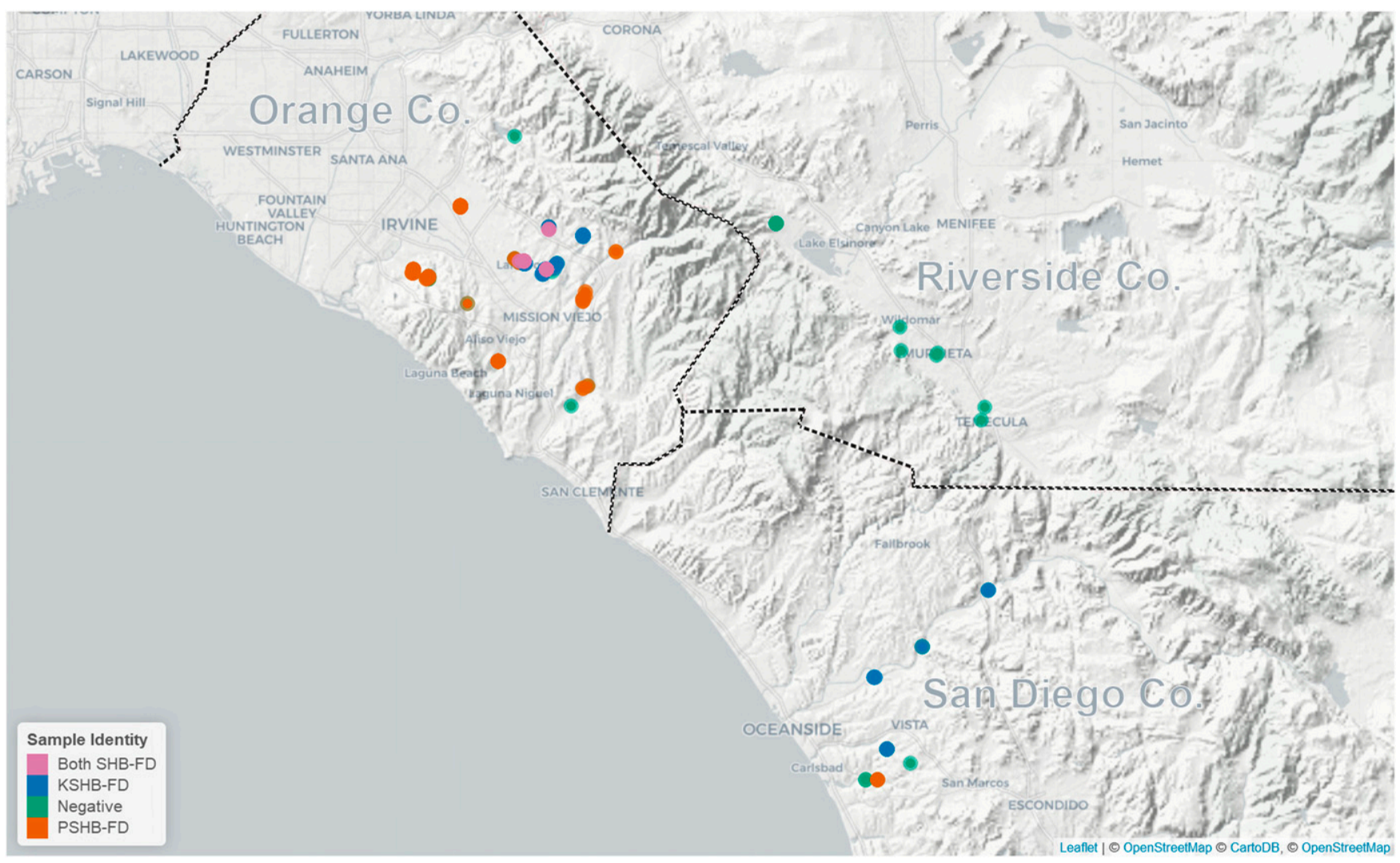

Fig. 4. Distribution of both polyphagous shot hole borer (PSHB) and Kuroshio shot hole borer (KSHB) from field sampling across 26 city park locations in Orange, Riverside, and San Diego Counties. Diagnosis of these pest-disease complexes was done through conventional fungal isolation and real-time quantitative PCR identification of recovered fungi. PSHB is mainly present in the Orange County locations, with a single positive location in San Diego County, whereas KSHB is present mainly in the San Diego locations; however, it is also present in close proximity to PSHB in Orange County. All points visited in Riverside were negative. The map was generated using R package "leaflet." FD, Fusarium dieback.

The invasion of Euwallacea spp. from Southeast Asia has occurred in many places around the world (CABI 2015; Eskalen et al. 2013; Hulcr and Stelinski 2017; O’Donnell et al. 2015; Paap et al. 2018; Short et al. 2017), with people potentially having a significant impact on moving this pest around unintentionally from infested to uninfested areas. This can happen through movement of infested nursery materials and firewood that may contain live beetles (Haack et al. 2010; Sanborn 1996). For instance, KSHB was positively identified in Santa Barbara from suspect Platanus racemosa wood using these assays (data not shown), which was unexpected, because KSHB had previously not been detected in the northern part of Orange County or anywhere in Los Angeles County. The beetles were also trapped and identified (RugmanJones and Stouthamer 2017) in this area as well as in San Luis Obispo. It is still unknown how KSHB spread into these areas undetected throughout Los Angeles County, because KSHB has concentrated populations south of Los Angeles County. Diagnosing these suspect samples and understanding the distribution of these pests will provide insight into their spread (Fig. 4), and they will provide useful information to inform land managers and the public to help prevent additional movement of infested material. Some potential pesticide treatment options have been researched, including injection and spray applications with insecticides, fungicides, and a biopesticide on sycamore in California (Mayorquin et al. 2018), with some found to be effective in reducing further infestation of the beetles compared with the controls. These provide arborists, nurseries, and land managers with options to prevent further infestation. Early detection can increase the survival of affected hosts while reducing the spread of these beetles into new areas by reducing their accumulation on hosts. Future studies should consider identifying both the beetles and their fungi in a given SHB-FD complex in one reaction if the hydrolysis probe combinations will permit it. Quantitative molecular approaches can aid in understanding the dynamics of SHB fungal mutualists and their function in the
SHB lifecycle as well as our ability to diagnose plants and manage infestations through early detection to prevent further spread of these invasive pests on regional and global scales.

\section{Acknowledgments}

We thank J. Pouzoulet for his advice on primer and probe design as well as E. Reyes, N. Riley, K. Moreno, D. Trannam, Z. Tan, and D. Vega for assistance in the laboratory and greenhouse. The funders had no role in study design, data collection and analysis, decision to publish, or preparation of the manuscript.

\section{Literature Cited}

Altschul, S. F., Gish, W., Miller, W., Myers, E. W., and Lipman, D. J. 1990. Basic local alignment search tool. J. Mol. Biol. 215:403-410.

Atallah, Z. K., Bae, J., Jansky, S. H., Rouse, D. I., and Stevenson, W. R. 2007. Multiplex real-time quantitative PCR to detect and quantify Verticillium dahliae colonization in potato lines that differ in response to Verticillium wilt. Phytopathology 97:865-872.

Beaver, R. A. 1989. Insect-fungus relationships in the bark and ambrosia beetles. Pages 121-143 in: Insect-Fungus Interactions. N. Wilding, N. M. Collins, P.M. Hammond, and J. F. Webber, eds. Academic Press, London.

Beaver, R. A., Sittichaya, W., and Liu, L. Y. 2014. A synopsis of the scolytine ambrosia beetles of Thailand (Coleoptera: Curculionidae: Scolytinae). Zootaxa 3875:1-82

Billones-Baaijens, R., Úrbez-Torres, J. R., Liu, M., Ayres, M., Sosnowski, M., and Savocchia, S. 2018. Molecular methods to detect and quantify Botryosphaeriaceae inocula associated with grapevine dieback in Australia. Plant Dis. 102:1489-1499.

Bodles, W. J., Fossdal, C. G., and Woodward, S. 2006. Multiplex real-time PCR detection of pathogen colonization in the bark and wood of Picea sitchensis clones differing in resistance to Heterobasidion annosum. Tree Physiol. 26: 775-782.

Boland, J. M. 2016. The impact of an invasive ambrosia beetle on the riparian habitats of the Tijuana River Valley, California. PeerJ 4:e2141.

Brancart, F., Rodriguez-Villalobos, H., Fonteyne, P. A., Peres-Bota, D., and Liesnard, C. 2005. Quantitative TaqMan PCR for detection of Pneumocystis jiroveci. J. Microbiol. Methods 61:381-387.

Bustin, S. A., Benes, V., Garson, J. A., Hellemans, J., Huggett, J., Kubista, M., Mueller, R., Nolan, T., Pfaffl, M. W., Shipley, G. L., and Vandesompele, J. 2009. The MIQE guidelines: minimum information for publication of quantitative real-time PCR experiments. Clin. Chem. 55:611-622. 
CABI. 2015. Euwallacea fornicatus Distribution Map. https://www.cabi.org/isc/ datasheet $/ 57163$

Carrillo, D., Duncan, R. E., Ploetz, J. N., Campbell, A. F., Ploetz, R. C., and Peña, J. E. 2014. Lateral transfer of a phytopathogenic symbiont among native and exotic ambrosia beetles. Plant Pathol. 63:54-62.

Chandelier, A., Massot, M., Fabreguettes, O., Gischer, F., Teng, F., and Robin, C. 2018. Early detection of Cryphonectria parasitica by real-time PCR. Eur. J. Plant Pathol. 153:135-152.

Cheng J, Karambelkar B, Xie Y. Leaflet: Create Interactive Web Maps with the JavaScript "Leaflet" Library [Internet]. 2017. Available: https://cran.r-project.org/ package $=$ leaflet

Cooperband, M. F., Stouthamer, R., Carrillo, D., Eskalen, A., Thibault, T., Cossé, A. A., Castrillo, L. A., Vandenberg, J. D., and Rugman-Jones, P. F. 2016. Biology of two members of the Euwallacea fornicatus species complex (Coleoptera: Curculionidae: Scolytinae), recently invasive in the USA, reared on an ambrosia beetle artificial diet. Agric. For. Entomol. 18:223-237.

Demeke, T., Ratnayaka, I., and Phan, A. 2009. Effects of DNA extraction and purification methods on real-time quantitative PCR analysis of Roundup Ready soybean. J. AOAC Int. 92:1136-1144.

Eskalen, A., Stouthamer, R., Lynch, S. C., Rugman-Jones, P. F., Twizeyimana, M., Gonzalez, A., and Thibault, T. 2013. Host range of fusarium dieback and its ambrosia beetle (Coleoptera: Scolytinae) vector in southern California. Plant Dis. 97:938-951.

Freeman, S., Sharon, M., Dori-Bachash, M., Maymon, M., Belausov, E., Maoz, Y., Margalit, O., Protasov, A., and Mendel, Z. 2016. Symbiotic association of three fungal species throughout the life cycle of the ambrosia beetle Euwallacea nr. fornicatus. Symbiosis 68:115-128.

Freeman, S., Sharon, M., Maymon, M., Mendel, Z., Protasov, A., Aoki, T., Eskalen, A., and O'Donnell, K. 2013. Fusarium euwallaceae sp. nov.-a symbiotic fungus of Euwallacea sp., an invasive ambrosia beetle in Israel and California. Mycologia 105:1595-1606.

Glass, N. L., and Donaldson, G. C. 1995. Development of primer sets designed for use with the PCR to amplify conserved genes from filamentous ascomycetes. Appl. Environ. Microbiol. 61:1323-1330.

Gomez, D. F., Skelton, J., Steininger, M. S., Stouthamer, R., Rugman-Jones, P., Sittichaya, W., Rabaglia, R. J., and Hulcr, J. 2018. Species delineation within the Euwallacea fornicatus (Coleoptera: Curculionidae) complex revealed by morphometric and phylogenetic analyses. Insect Syst. Diver. 2:1-11.

Haack, R. A., Petrice, T. R., and Wiedenhoeft, A. C. 2010. Incidence of bark-and wood-boring insects in firewood: a survey at Michigan's Mackinac Bridge. J. Econ. Entomol. 103:1682-1692.

Heller, W. P., and Keith, L. M. 2018. Real-time PCR assays to detect and distinguish the rapid 'Ōhi'a death pathogens Ceratocystis lukuohia and C. huliohia. Phytopathology 108:1395-1401.

Hofstetter, R. W., Dinkins-Bookwalter, J., Davis, T. S., and Klepzig, K. D. 2015. Symbiotic, associations of bark beetles. Pages 209-245 in: Bark Beetles. F. E. Vega and R. W. Hofstetter, eds. Academic Press, San Diego, CA.

Hothorn, T., Bretz, F., and Westfall, P. 2008. Simultaneous inference in general parametric models. Biometrical J. 50:346-363.

Hulcr, J., and Cognato, A. I. 2010. Repeated evolution of crop theft in fungusfarming ambrosia beetles. Evolution 64:3205-3212.

Hulcr, J., and Dunn, R. R. 2011. The sudden emergence of pathogenicity in insectfungus symbioses threatens naive forest ecosystems. Proc. R. Soc. Lond. [Biol.] 278:2866-2873.

Hulcr, J., and Stelinski, L. L. 2017. The ambrosia symbiosis: from evolutionary ecology to, practical management. Annu. Rev. Entomol. 62:285-303.

Kasson, M. T., O’Donnell, K., Rooney, A. P., Sink, S., Ploetz, R. C., Ploetz, J. N., Konkol, J. L., Carrillo, D., Freeman, S., Mendel, Z., Smith, J. A., Black, A. W., Hulcr, J., Bateman, C., Stefkova, K., Campbell, P. R., Geering, A. D. W., Dann, E. K., Eskalen, A., Mohotti, K., Short, D. P. G., Aoki, T., Fenstermacher, K. A., Davis, D. D., and Geiser, D. M. 2013. An inordinate fondness for Fusarium: phylogenetic diversity of fusaria cultivated by ambrosia beetles in the genus Euwallacea on avocado and other plant hosts. Fungal Genet. Biol. 56:147-157.

Kleiber, C., and Zeileis, A. 2015. Package 'AER'. R package version 1.2-4, https://cran.r-project.org/web/packages/AER/AER.pdf.

Koressaar, T., and Remm, M. 2007. Enhancements and modifications of primer design program Primer3. Bioinformatics 23:1289-1291.

Kostovcik, M., Bateman, C. C., Kolarik, M., Stelinski, L. L., Jordal, B. H., and Hulcr, J. 2015. The ambrosia symbiosis is specific in some species and promiscuous in others: evidence from community pyrosequencing. ISME J. 9:126-138

Li, W., Abad, J. A., French-Monar, R. D., Rascoe, J., Wen, A., Gudmestad, N. C., Secor, G. A., Lee, M., Duan, Y., and Levy, L. 2009. Multiplex real-time PCR for detection, identification and quantification of 'Candidatus Liberibacter solanacearum'in potato plants with zebra chip. J. Microbiol. Methods 78: 59-65.

Li, W., Hartung, J. S., and Levy, L. 2006. Quantitative real-time PCR for detection and identification of Candidatus Liberibacterspecies associated with citrus huanglongbing. J. Microbiol. Methods 66:104-115.

Lynch, S. C., Twizeyimana, M., Mayorquin, J. S., Wang, D. H., Na, F., Kayim, M., Kasson, M. T., Thu, P. Q., Bateman, C., Rugman-Jones, P., and Hulcr, J. 2016. Identification, pathogenicity and abundance of Paracremonium pembeum sp. nov. and Graphium euwallaceae sp. nov.- two newly discovered mycangial associates of the polyphagous shot hole borer (Euwallacea sp.) in California. Mycologia 108:313-329.

Mayorquin, J. S., Carrillo, J. D., Twizeyimana, M., Peacock, B. B., Sugino, K. Y., Na, F., Wang, D. H., Kabashima, J. N., and Eskalen, A. 2018. Chemical management of invasive shot hole borer and Fusarium dieback in California sycamore (Platanus racemosa) in Southern California. Plant Dis. 102: $1307-1315$

Mendel, Z., Protasov, A., Sharon, M., Zveibil, A., Yehuda, S. B., O’Donnell, K. Rabaglia, R., Wysoki, M., and Freeman, S. 2012. An Asian ambrosia beetle Euwallacea fornicatus and its novel symbiotic fungus Fusarium sp. pose a serious threat to the Israeli avocado industry. Phytoparasitica 40: 235-238.

Na, F., Carrillo, J. D., Mayorquin, J. S., Ndinga-Muniania, C., Stajich, J. E. Stouthamer, R., Huang, Y. T., Lin, Y. T., Chen, C. Y., and Eskalen, A. 2018. Two novel fungal symbionts Fusarium kuroshium sp. nov. and Graphium kuroshium sp. nov. of Kuroshio shot hole borer (Euwallacea sp. nr. fornicatus) cause Fusarium dieback on woody host species in California. Plant Dis. 102:1154-1164.

Nolan, T., Hands, R. E., Ogunkolade, W., and Bustin, S. A. 2006. SPUD: a quantitative PCR assay for the detection of inhibitors in nucleic acid preparations. Anal. Biochem. 351:308-310.

OC Parks. 2017. Shot Hole Borer: Managing the Invasive Beetle. https://oc-parks-gis maps.arcgis.com/apps/Cascade/index.html?appid=680fd0c9e73f4857a8477791f7ee796f O’Donnell, K., Libeskind-Hadas, R., Hulcr, J., Bateman, C., Kasson, M. T., Ploetz, R. C., Konkol, J. L., Ploetz, J. N., Carrillo, D., Campbell, A., and Duncan, R. E. 2016. Invasive Asian Fusarium-Euwallacea ambrosia beetle mutualists pose a serious threat to forests, urban landscapes and the avocado industry. Phytoparasitica 44:435-442.

O’Donnell, K., Sink, S., Libeskind-Hadas, R., Hulcr, J., Kasson, M. T., Ploetz, R. C., Konkol, J. L., Ploetz, J. N., Carrillo, D., Campbell, A., Duncan, R. E. Liyanage, P. N., Eskalen, A., Na, F., Geiser, D. M., Bateman, C., Freeman, S., Mendel, Z., Sharon, M., Aoki, T., Crossé, A. A., and Rooney, A. P. 2015 Discordant phylogenies suggest repeated host shifts in the FusariumEuwallacea ambrosia beetle mutualism. Fungal Genet. Biol. 82:277-290.

Oliveira, A. C., Vallim, M. A., Semighini, C. P., Araújo, W. L., Goldman, G. H., and Machado, M. A. 2002. Quantification of Xylella fastidiosa from citrus trees by real-time polymerase chain reaction assay. Phytopathology 92 1048-1054

Osman, F., and Rowhani, A. 2006. Application of a spotting sample preparation technique for the detection of pathogens in woody plants by RT-PCR and real-time PCR (TaqMan). J. Virol. Methods 133:130-136.

Paap, T., De Beer, Z. W., Migliorini, D., Nel, W. J., and Wingfield, M. J. 2018. The polyphagous shot hole borer (PSHB) and its fungal symbiont Fusarium euwallaceae: a new invasion in South Africa. Australas. Plant Pathol. 47 231-237.

Palacio-Bielsa, A., Cambra, M. A., and López, M. M. 2009. PCR detection and identification of plant-pathogenic bacteria: updated review of protocols (1989-2007). J. Plant Pathol. 91:249-297.

Pearson, W. R. 2016. Finding protein and nucleotide similarities with FASTA. Curr. Protoc. Bioinformatics 53:3-9.

Ploetz, R. C., Hulcr, J., Wingfield, M. J., and De Beer, Z. W. 2013. Destructive tree diseases associated with ambrosia and bark beetles: black swan events in tree pathology? Plant Dis. 97:856-872.

Postollec, F., Falentin, H., Pavan, S., Combrisson, J., and Sohier, D. 2011. Recent advances in quantitative PCR (qPCR) applications in food microbiology. Food Microbiol. 28:848-861

Pouzoulet, J., Mailhac, N., Couderc, C., Besson, X., Daydé, J., Lummerzheim, M., and Jacques, A. 2013. A method to detect and quantify Phaeomoniella chlamydospora and Phaeoacremonium aleophilum DNA in grapevine-wood samples. Appl. Microbiol. Biotechnol. 97:10163-10175.

Qu, X. S., Wanner, L. A., and Christ, B. J. 2011. Multiplex real-time PCR (TaqMan) assay for the simultaneous detection and discrimination of potato powdery and common scab diseases and pathogens. J. Appl. Microbiol. 110: 769-777.

Raso, A., and Biassoni, R. 2014. Twenty years of qPCR: a mature technology? Pages 1-3 in: Quantitative Real-Time PCR. R. Biassoni and A. Raso, eds. Springer, New York, NY.

R Development Core Team. 2013. R: A Language and Environment for Statistical Computing. R Foundation for Statistical Computing, Vienna, Austria.

Rugman-Jones, P. F., and Stouthamer, R. 2017. High-resolution melt analysis without DNA extraction affords rapid genotype resolution and species identification. Mol. Ecol. Resour. 17:598-607.

Sanborn, S. R. 1996. Controlling bark beetles in wood residue and firewood. Tree Notes 3. California Department of Forestry and Fire Protection, Sacramento, CA.

Schaad, N. W., and Frederick, R. D. 2002. Real-time PCR and its application for rapid plant disease diagnostics. Can. J. Plant Pathol. 24:250-258.

Schoch, C. L., Seifert, K. A., Huhndorf, S., Robert, V., Spouge, J. L., Levesque, C. A., Chen, W., Bolchacova, E., Voigt, K., Crous, P. W., and Miller, A. N 2012. Nuclear ribosomal internal transcribed spacer (ITS) region as a universal DNA barcode marker for Fungi. P. Natl. Acad. Sci. 109:6241-6246. Short, D. P., O’Donnell, K., Stajich, J. E., Hulcr, J., Kijimoto, T., Berger, M. C. Macias, A. M., Spahr, E. J., Bateman, C. C., Eskalen, A., and Lynch, S. C. 
2017. PCR multiplexes discriminate Fusarium symbionts of invasive Euwallacea ambrosia beetles that inflict damage on numerous tree species throughout the United States. Plant Dis. 101:233-240.

Stouthamer, R., Rugman-Jones, P., Thu, P. Q., Eskalen, A., Thibault, T., Hulcr, J., Wang, L. J., Jordal, B. H., Chen, C. Y., Cooperband, M., and Lin, C. S. 2017. Tracing the origin of a cryptic invader: phylogeography of the Euwallacea fornicatus (Coleoptera: Curculionidae: Scolytinae) species complex. Agric. For. Entomol. 19:366-375.

Tellenbach, C., Grünig, C. R., and Sieber, T. N. 2010. Suitability of quantitative real-time PCR to estimate the biomass of fungal root endophytes. Appl. Environ. Microbiol. 76:5764-5772.

Thompson, J. D., Gibson, T. J., Plewniak, F., Jeanmougin, F., and Higgins, D. G. 1997. The CLUSTAL X windows interface: flexible strategies for multiple sequence alignment aided by quality analysis tools. Nucleic Acids Res. 25: 4876-4882.
Untergasser, A., Cutcutache, I., Koressaar, T., Ye, J., Faircloth, B. C., Remm, M., and Rozen, S. G. 2012. Primer3 - new capabilities and interfaces. Nucleic Acids Res. 40:e115.

Venables, W. N., and Ripley, B. D. 2002. Tree-based methods. Pages 251-269 in: Modern Applied Statistics with S. J. Chambers, W. Eddy, W. Härdle, S. Sheather, and L. Tierney, eds. Springer, New York, NY.

White, T. J., Bruns, T., Lee, S., and Taylor, J. 1990. Amplification and direct sequencing of fungal ribosomal RNA genes for phylogenetics. Pages 315-322 in: PCR Protocols: A Guide to Methods and Application. M. A. Innis, D. H. Gelfand, J. J. Sninsky, and T. J. White, eds. Academic Press, New York, NY.

Wickham, H. 2011. The split-apply-combine strategy for data analysis. J. Stat Softw. 40:1-29.

Wickham, H. 2016. ggplot2: elegant graphics for data analysis. Springer, London.

Zitnick-Anderson, K., Simons, K., and Pasche, J. S. 2018. Detection and qPCR quantification of seven Fusarium species associated with the root rot complex in field pea. Can. J. Plant Pathol. 40:261-271. 